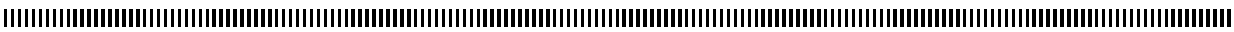

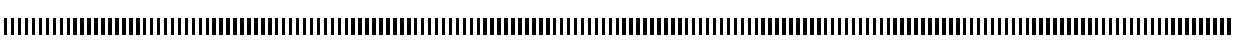

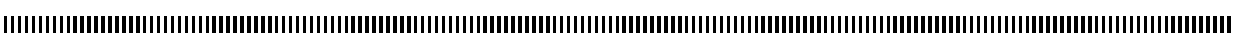

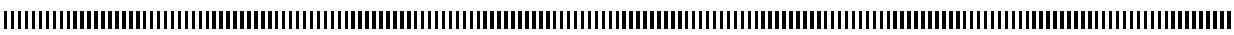

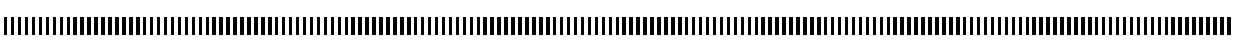

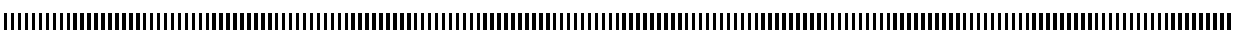

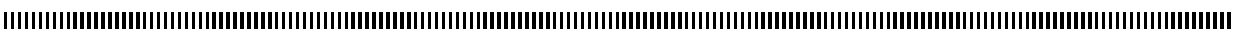

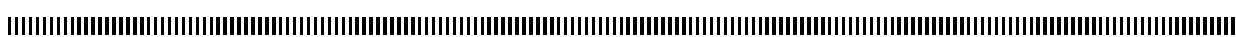
|||||||||||||||||||||||||||||||||||||||||||||||||||||||||||||||||||||||||||||||||||||||||||||||||||||||||||||||||||||||||||||||||||||||||||||||||||||||||||||||||||||||||||||||||||||||||||||||||||||

\title{
Commande optimale en temps minimal d'un procédé biologique d'épuration de l'eau
}

Walid Bouhafs ${ }^{a}$ — Nahla Abdellatif b,a — Frédéric Jean c — Jérôme Harmand d,e

a Laboratoire de Modélisation Mathématique et Numérique dans les Sciences de l'Ingénieur (LAMSIN), Ecole Nationale d'Ingénieurs de Tunis (ENIT), BP 37, Le Belvédère 1002 Tunis, Tunisie.

b Ecole Nationale des Sciences de l'Informatique, Université de Manouba Campus Universitaire, 2010, Manouba, Tunisie.

c ENSTA, UMA, 828, Boulevard des Maréchaux, 91762 Palaiseau Cedex, France.

d INRA, UR0050, Laboratoire de Biotechnologie de l'Environnement,

Avenue des Etangs, 11100 Narbonne, France.

e Projet INRIA-INRA MODEMIC, UMR MISTEA, Laboratoire de Mathématiques, Informatique et Statistique pour l'Environnement et l'Agronomie,

2, place Pierre Viala, 34060 Montpellier, France.

|

RÉSUMÉ. Dans ce travail, on considère un problème de contrôle optimal d'un procédé biologique séquentiel discontinu pour le traitement de la pollution. Ce modèle fait intervenir deux réactions biologiques, l'une étant aérobie et l'autre anoxique. On s'intéresse, dans un premier temps, à un problème de contrôle optimal en temps minimal puis en temps et en énergie. On prouve l'existence de trajectoires optimales et on calcule, dans chaque cas identifié, les contrôles optimaux correspondants.

ABSTRACT. In this work, we consider an optimal control problem of a biological sequencing batch reactor for the treatment of pollutants. This model includes two biological reactions, one being aerobic while the other is anoxic. We are first interested in a problem of optimal control in time and then, in both time and energy. The existence of the optimal trajectories is proven and the corresponding optimal controls are derived in each case.

MOTS-CLÉS : Contrôle optimal, réacteur biologique séquentiel discontinu, traitement de l'eau.

KEYWORDS : Optimal control, sequencing batch reactor, water treatment. 


\section{Introduction}

De part leur simplicité de mise en oeuvre, les systèmes biologiques sont aujourd'hui les procédés les plus utilisés pour le traitement des eaux. Le traitement biologique des polluants organiques et/ou chimiques contenus dans ces eaux usées consiste en la transformation de la matière biodégradable en boues (également appelée biomasse qui se trouve sous forme solide dans le système). Le principe du traitement biologique est de mettre en contact des micro-organismes et les polluants dans des enceintes (réacteurs) dans lesquelles il est possible de contrôler les conditions environnementales. Dans une seconde enceinte, appelée décanteur ou clarificateur, les boues et l'eau épurée sont séparés. En l'absence d'agitation, les bactéries s'agglomèrent en flocs qui, plus denses que l'eau, décantent sous l'effet de la gravité. Les boues sont recyclées ou éliminées alors que l'eau traitée est rejetée dans l'environnement. Plusieurs technologies permettent de mettre en oeuvre ces principes de traitement et de séparation pour l'épuration des eaux usées. Parmi ces technologies, les réacteurs séquentiels discontinus (SBR pour Sequencing Batch Reactors) présentent certains avantages par rapport à leur homologues continus. En séparant dans le temps plutôt que dans l'espace les phases de traitement et de séparation évoquées ci-dessus, les SBR permettent notamment un meilleur contrôle du procédé et donc de la qualité des eaux rejetées. Le prix à payer est que le système considéré fonctionne maintenant en mode batch et non plus en continu. Il nécessite donc la mise en place en amont d'un bassin de stockage des eaux usées qui arrivent, elles, continument à la station. Dans ce cas, un problème est de minimiser le temps pendant lequel le réacteur de traitement est indisponible, c'est-à-dire le temps de réaction nécessaire au traitement des eaux si on suppose le temps de décantation constant. Plusieurs travaux se sont intéressés à la mise au point d'algorithmes de contrôle optimal en temps minimal des SBR ([1],[2]), et, en particulier, d'une stratégie permettant de déterminer les instants de commutation entre phases aérobie et anoxique, pour minimiser le temps total de réaction ([3]). Pour des raisons techniques, il n'avait pas été possible dans les travaux de Mazouni ([1],[3]) d'envisager de moduler la concentration en oxygène et seules des situations purement aérobie (saturation de la concentration en oxygène) et purement anoxique (pas d'injection d'oxygène) avaient été modélisées. Or, des systèmes de régulation fine de la concentration en oxygène sont aujourd'hui disponibles sur le marché. On se propose donc de revisiter ce problème de contrôle optimal en temps minimal des SBR dans un contexte où la concentration en oxygène serait maintenue suffisamment faible pour autoriser les réactions aérobies et anoxiques de se réaliser simultanément. Cette simultanéité de réactions est classique dans les procédés à boues activées et bien modélisée dans les modèles ASM de l'IWA ([4]). Par suite, il s'agit de rechercher dans quelle mesure une telle approche de modulation de la concentration en oxygène pourrait être meilleure que la solution par alternances de phases proposée dans [3]. Le problème ainsi posé nécessite de considérer un modèle en 3 dimensions, modèle pour lequel le problème de commande est très difficile à résoudre directement. C'est la raison pour laquelle on se propose d'étudier dans un premier temps un problème légèrement différent où n'apparaît pas explicitement de couplage entre les substrats alors que c'était le cas dans le travail de D. Mazouni ([1],[3]). Plus spécifiquement, nous nous intéressons à un modèle dans lequel la concentration en oxygène est la variable de contrôle et où deux réactions biologiques - pouvant avoir lieu simultanément - sont nécessaires pour traiter deux types de substrats distincts. Les deux biomasses microbiennes en jeu sont respectivement limitées et inhibées par leur substrat respectif, l'une étant favorisée par l'oxygène (réaction aérobie) alors que la seconde est 
inhibée (réaction anoxique). Sur le plan pratique, on simplifie donc le cas considéré par D. Mazouni ([1],[3]) au sens où les processus de nitrification et de dénitrification sont bien contrôlés par l'oxygène mais où la contrainte sur la matière organique nécessaire à la dénitrification est ignorée. Cette simplification nous permet de traiter ce cas dans deux dimensions et de résoudre le problème de contrôle optimal en temps et en énergie. Nous avons de bonnes raisons de penser que les résultats de cette première étude nous aiderons à aborder le problème en trois dimensions où les variables sont couplées par un substrat supplémentaire (le carbone). Dans la première partie de l'article, on s'intéresse à la mise au point d'une stratégie de contrôle optimal en temps minimal, puis, dans une seconde partie, à la synthèse d'une commande optimale en temps et en énergie, afin de minimiser à la fois le temps de réaction et la quantité d'oxygène consommé.

\section{Modélisation}

On considère les réactions biologiques suivantes :

$$
\begin{gathered}
s_{1}+u \rightarrow x_{1} \\
s_{2}+u \rightarrow x_{2}
\end{gathered}
$$

où $s_{1}$ et $s_{2}$ désignent les substrats que l'on cherche à éliminer, $x_{1}$ et $x_{2}$ sont les biomasses, c'est-à-dire les concentrations en bactéries qui consomment respectivement $s_{1}$ et $s_{2}$ pour leur croissance.

La première réaction est une réaction favorisée par la présence d'oxygène tandis que la seconde est inhibée par l'oxygène.

La concentration en oxygène $u$ est considérée comme la commande dans ce système. En supposant que l'on s'intéresse à un système fonctionnant en mode batch, le modèle du bioprocédé s'écrit :

$$
\left\{\begin{array}{l}
\dot{x}_{1}=\mu_{1}\left(s_{1}, u\right) x_{1}, \\
\dot{s}_{1}=-\mu_{1}\left(s_{1}, u\right) x_{1}, \\
\dot{x}_{2}=\mu_{2}\left(s_{2}, u\right) x_{2}, \\
\dot{s}_{2}=-\mu_{2}\left(s_{2}, u\right) x_{2},
\end{array}\right.
$$

où la fonction de croissance $\mu_{1}$ est strictement croissante par rapport à $u$ et la fonction $\mu_{2}$ est strictement décroissante par rapport à $u$.

Comme la première réaction est une réaction aérobie, la variation de $s_{1}$ est nulle si l'oxygène est absent. De plus, cette variation augmente d'une manière continue lorsque la quantité de l'oxygène injectée dans le réacteur augmente aussi. Et inversement pour la variation de $s_{2}$, ce qui nous conduit à supposer :

$\mathbf{H}_{1}: \mu_{1}\left(s_{1}, u\right)=f_{1}^{0}\left(s_{1}\right) \mu(u)$ et $\mu_{2}\left(s_{2}, u\right)=f_{2}^{0}\left(s_{2}\right)(K-\mu(u))$, avec $K>0, f_{1}^{0}$ et $f_{2}^{0}$ deux fonctions positives, bornées, de classe $C^{1}$ et qui vérifient $f_{1}^{0}(0)=f_{2}^{0}(0)=0$. 
$\mu$ est une fonction positive, de classe $C^{1}$, strictement croissante et vérifie $\mu(0)=0$ et $\left.\mu\left(u_{\max }\right)=K, u_{\max } \in\right] 0,+\infty[$.

$\mathbf{H}_{2}: 0 \leq u(t) \leq u_{\max }$

Notons que l'on a, par conservation de masse dans le système $(2.3): \dot{s}_{i}(t)+\dot{x}_{i}(t)=0$ pour $i=1,2$, pour tout $t$ positif. On pose alors $M_{1}=s_{1}(0)+x_{1}(0)$ et $M_{2}=s_{2}(0)+x_{2}(0)$. Sans perte de généralité, supposons que $M_{1}=M_{2}=1$.

Le système (2.3) est alors équivalent au système bidimensionnel :

$$
\left\{\begin{array}{l}
\dot{s}_{1}=-\mu(u) f_{1}\left(s_{1}\right), \\
\dot{s}_{2}=-(K-\mu(u)) f_{2}\left(s_{2}\right),
\end{array}\right.
$$

où $f_{i}\left(s_{i}\right)=f_{i}^{0}\left(s_{i}\right)\left(1-s_{i}\right), i=1,2$. Les fonctions $f_{1}$ et $f_{2}$ sont positives, bornées, de classe $C^{1}$ et vérifient $f_{1}(0)=f_{2}(0)=0$ et $f_{1}(1)=f_{2}(1)=0$. Autrement dit, $f_{1}$ et $f_{2}$ sont nulles en absence de substrat ou de biomasse associée.

Le but est de régler la concentration d'oxygène $u$ afin d'amener les concentrations $s_{1}$ et $s_{2}$ en dessous d'un certain seuil.

\section{Problème de contrôle en temps minimal}

Le premier problème que l'on considère est d'amener en temps minimal les concentrations $s_{1}$ et $s_{2}$ en dessous de seuils spécifiés par l'utilisateur. Cet objectif se formule comme un problème de contrôle optimal.

\subsection{Spécification du problème}

On considère le problème de contrôle optimal suivant :

$$
(\mathcal{P})\left\{\begin{array}{l}
\text { Minimiser } t_{f}, \\
u \in L^{\infty}\left(\left[0, t_{f}\right],\left[0, u_{\max }\right]\right), \\
s \text { solution de }(2.4) \text { tel que } s(0)=s^{0} \text { et } s\left(t_{f}\right) \in \mathcal{C} .
\end{array}\right.
$$

où $\mathcal{C}=[0, a] \times[0, b]$ et $(a, b) \in] 0,1[2$. Nous nous intéressons aux trajectoires qui partent de $\left.s^{0} \in\right] 0,1\left[^{2}\right.$ (les concentrations initiales des substrats) et qui atteignent la cible $(\mathcal{C})$ (les concentrations souhaitées) en un temps minimal.

\subsection{Solutions optimales}

On commence par énoncer les deux résultats suivants :

Proposition 3.1 Pour tout $\left.s^{0} \in\right] 0,1\left[2\right.$, il existe un contrôle $u \in L^{\infty}\left(\left[0, t_{f}\right],\left[0, u_{\max }\right]\right)$ tel que la trajectoire $s_{u}($.$) relie s^{0}$ à la cible $\mathcal{C}$. 
Preuve. Si $s^{0}$ appartient à l'intérieur de la cible, la proposition est triviale. Si $s^{0}$ est à l'extérieur de la cible, il suffit de considérer une trajectoire donnée par l'union de deux segments : un segment horizontal qui correspond au contrôle $u=u_{\max }$, ce segment part de $s^{0}$ et arrive à l'axe $\left\{s_{1}=a\right\}$, et un segment vertical qui correspond au contrôle $u=0$. Ce segment part du point qui appartient à l'intersection de l'axe $\left\{s_{1}=a\right\}$ et du premier segment et arrive à la cible $\mathcal{C}$.

Proposition 3.2 Pour toute condition initiale $\left.s^{0} \in\right] 0,1\left[^{2}\right.$, il existe une solution au problème $(\mathcal{P})$.

Preuve. On démontre ce résultat en appliquant un théorème de Filippov (voir par exemple [5, théorème 6.2.1]). Remarquons d'abord que, comme $f_{1}$ et $f_{2}$ s'annulent en 0 et en 1 , toute trajectoire $s(\cdot)$ issue de $\left.s^{0} \in\right] 0,1\left[^{2}\right.$ vérifie $s_{1}(t)$ et $\left.s_{2}(t) \in\right] 0,1\left[\right.$ pour tout $t \in\left[0, t_{f}\right]$ et est donc uniformément bornée. D'autre part, pour tout $(t, s) \in \mathbb{R}_{+} \times \mathbb{R}^{2}$, l'ensemble des vecteurs vitesse augmentés :

$$
V(t, s)=\left\{1,\left(\mu(u) f_{1}\left(s_{1}(t)\right),(K-\mu(u)) f_{2}\left(s_{2}(t)\right), u \in\left[0, u_{\max }\right]\right\}\right.
$$

est convexe et compact . Ainsi les hypothèses du théorème sont satisfaites et il existe un contrôle optimal $u$ tel que la trajectoire $s_{u}($.$) joint s_{0}$ à la cible $\mathcal{C}$ en temps minimal.

Pour déterminer les trajectoires optimales, nous décomposons le domaine complémentaire de la cible $\mathcal{C}$ dans $\left[0,1\left[\times\left[0,1\right.\right.\right.$ [ en trois régions. Soient $D_{1}, D_{2}$ et $D_{3}$ les trois domaines disjoints définis par : $D_{1}=\left[0, a\left[\times\left[b, 1\left[, D_{2}=\left[a, 1\left[\times\left[b, 1\left[\right.\right.\right.\right.\right.\right.\right.\right.$, et $D_{3}=[a, 1[\times[0, b[$.

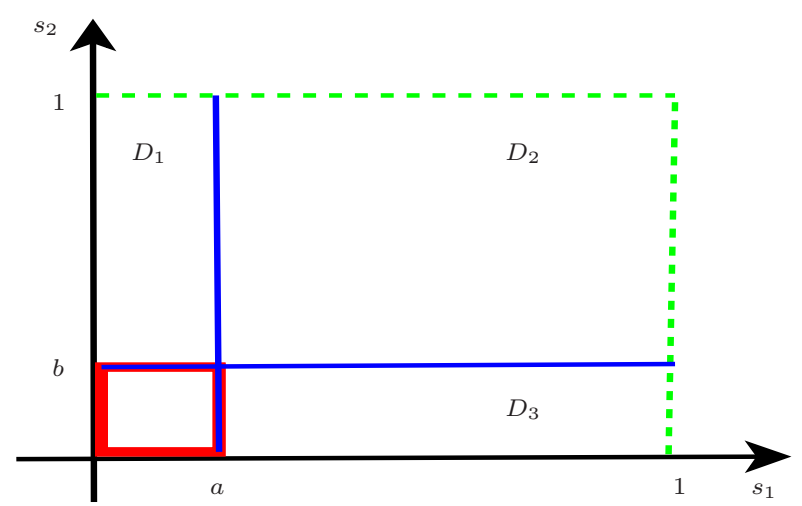

Figure 1. Partition du domaine

Nous établissons, maintenant, une description complète de la synthèse optimale du problème $(\mathcal{P})$.

Théorème 3.1 Pour une condition initiale $\left.s^{0} \in\right] 0,1\left[{ }^{2}\right.$, les solutions du problème $(\mathcal{P})$ sont les suivantes.

1) Si s ${ }^{0} \in D_{1}$, la trajectoire optimale du problème $(\mathcal{P})$ est unique. C'est le segment vertical qui part de $s^{0}$.

2) Si $s^{0} \in D_{3}$, la trajectoire optimale du problème $(\mathcal{P})$ est unique. C'est le segment horizontal qui part de $s^{0}$. 
3) Si $s^{0} \in D_{2}$, il existe une infinité de trajectoires optimales pour le problème $(\mathcal{P})$. Ce sont toutes les trajectoires du système issues de $s^{0}$ qui touchent la cible au point $\bar{s}=(a, b)$ (coin de la cible). De plus, le temps minimal est donné par :

$$
t_{f}=\frac{1}{K}\left(\int_{a}^{s_{1}^{0}} \frac{d s}{f_{1}(s)}+\int_{b}^{s_{2}^{0}} \frac{d s}{f_{2}(s)}\right) .
$$

Preuve. On va appliquer le Principe du Maximum de Pontryagin [6, 7]. Le hamiltonien du problème $(\mathcal{P})$ est donné par :

$$
H(s, p, u)=-p_{1} \mu(u) f_{1}\left(s_{1}\right)-p_{2}(K-\mu(u)) f_{2}\left(s_{2}\right)+1,
$$

où $p=\left(p_{1}, p_{2}\right)$ est le vecteur adjoint. Si $(s(),. p()$.$) est une extrémale, alors$

$$
\left\{\begin{array}{l}
\dot{p_{1}}=-\frac{\partial H}{\partial s_{1}}=p_{1} \mu(u) f_{1}^{\prime}\left(s_{1}\right) \\
\dot{p_{2}}=-\frac{\partial H}{\partial s_{2}}=p_{2}(K-\mu(u)) f_{2}^{\prime}\left(s_{2}\right)
\end{array}\right.
$$

On a donc, pour $\bar{t} \in\left[0, t_{f}\right]$,

$$
p_{1}(t)=p_{1}(\bar{t}) \exp \left(\int_{\bar{t}}^{t} \mu(u(s)) f_{1}^{\prime}\left(s_{1}(s)\right) d s\right)
$$

et

$$
p_{2}(t)=p_{2}(\bar{t}) \exp \left(\int_{\bar{t}}^{t}(K-\mu(u(s))) f_{2}^{\prime}\left(s_{2}(s)\right) d s\right) .
$$

La condition de minimisation s'écrit : pour tout $t \in\left[0, t_{f}\right]$,

$$
H(s(t), p(t), u(t))=\min _{v \in \Omega} H(s(t), p(t), v),
$$

où $\Omega=\left[0, u_{\max }\right]$ est l'ensemble des valeurs du contrôle.

Posons : $s(t)=\left(s_{1}(t), s_{2}(t)\right), p(t)=\left(p_{1}(t), p_{2}(t)\right)$ et

$$
\varphi(t)=\frac{\partial H}{\partial u}(s(t), p(t), u(t))=\mu^{\prime}(u(t))\left(-p_{1}(t) f_{1}\left(s_{1}(t)\right)+p_{2}(t) f_{2}\left(s_{2}(t)\right)\right) .
$$

D'après l'équation (3.2) on déduit :

$-\operatorname{Si} \varphi(t)>0$ alors $u(t)=0$.

$-\operatorname{Si} \varphi(t)<0$ alors $u(t)=u_{\max }$.

- Si $\varphi(t)=0$ alors $u(t) \in] 0, u_{\max }[$.

De plus, la condition de transversalité suivante doit être satisfaite :

$$
\left\langle p\left(t_{f}\right),\left(y-s\left(t_{f}\right)\right)\right\rangle \leq 0 \text { pour tout } y \in \mathcal{C} .
$$

Montrons les trois points du théorème.

Soit $s^{0} \in D_{1}$. Considérons une trajectoire optimale $s(\cdot)$ issue de $s^{0}$ et le vecteur adjoint associé $p(\cdot)$. D'après le système $(2.4)$, on a $\dot{s}_{1} \leq 0$ et par conséquent $s_{1}<a$. Ceci implique que la trajectoire $s(\cdot)$ ne sort pas de la région $D_{1}$. Par suite, cette trajectoire touche 
la cible au point $s\left(t_{f}\right) \in\left\{s_{2}=b\right\}$ avec $\left.s_{1}\left(t_{f}\right) \in\right] 0, a[$. Dans ce cas, la condition de transversalité s'écrit : $p_{1}\left(t_{f}\right)=0$ et $p_{2}\left(t_{f}\right)>0$. D'où, $p_{1} \equiv 0$ et $p_{2}(t)>0$, pour tout $t \in\left[0, t_{f}\right]$. Par suite, le hamiltonien atteint son minimum en $u=0$ et la trajectoire correspondante à ce contrôle vérifie $\dot{s}_{1}=0$ et $\dot{s}_{2}<0$. Ceci montre le point 1 ) du théorème. Le point 2) se démontre de la même façon que 1).

Soit $s^{0} \in D_{2}$. Alors toute trajectoire optimale $s(\cdot)$ issue de $s^{0}$ touche la cible par le coin. En effet, si $s(\cdot)$ atteint la cible en un point $s\left(t_{f}\right)$ qui appartient à $\left\{s_{2}=b\right\} \cap D_{1}$, la trajectoire $s(\cdot)$ doit être un segment vertical inclus dans $D_{1}$. Ceci est absurde puisque $s_{0} \in D_{2}$. On raisonne de la même façon si la trajectoire atteint la cible en $s\left(t_{f}\right) \in D_{3}$.

Montrons que le temps nécessaire pour joindre $s^{0}$ au coin de la cible $\mathcal{C}$ ne dépend pas du contrôle $u$. En effet, du système $(2.4)$ on déduit que :

$$
\mu(u)=-\frac{\dot{s}_{1}}{f_{1}\left(s_{1}\right)} \quad \text { et } \quad K-\mu(u)=-\frac{\dot{s}_{2}}{f_{2}\left(s_{2}\right)} .
$$

En faisant la somme des deux équations de (3.3), on obtient :

$$
-K=\frac{\dot{s}_{1}}{f_{1}\left(s_{1}\right)}+\frac{\dot{s}_{2}}{f_{2}\left(s_{2}\right)} .
$$

En intégrant (3.4) par rapport au temps entre 0 et $t_{f}$, on déduit après un changement de variable que :

$$
t_{f}=\frac{1}{K}\left(\int_{a}^{s_{1}^{0}} \frac{d s}{f_{1}(s)}+\int_{b}^{s_{2}^{0}} \frac{d s}{f_{2}(s)}\right) .
$$

Donc toutes les trajectoires qui relient $s^{0}$ au coin de la cible $\mathcal{C}$ atteignent cette dernière en un temps qui ne dépend pas de $u$.

Remarque 3.1 Les zones $D_{1}$ et $D_{3}$ correspondent à des problèmes aérobie et anoxie stricts, respectivement, et cet aspect de réactions simultanées n'est en fait pas véritablement considéré. Seul le cas de la zone $D_{2}$ peut conduire à des réactions simultanées.

Remarquons aussi que si la cible est a priori petite (i.e. les seuils $a$ et $b$ sont petits), alors la condition initiale est quasiment toujours dans $D_{2}$. Quand la condition initiale est dans $D_{2}$, il y a une infinité de trajectoires optimales (toutes atteignent le coin). D'où la question supplémentaire : laquelle choisir? selon quel critère ? C'est la raison pour laquelle on définit maintenant le problème $(\mathcal{Q})$ qui s'énonce comme suit :

$$
(\mathcal{Q})\left\{\begin{array}{l}
\text { Minimiser } \int_{0}^{t_{f}} u^{2}(s) d s, \\
u \in L^{\infty}\left(\left[0, t_{f}\right],\left[0, u_{\max }\right]\right), \\
s \text { solution de }(2.4) \text { tel que } s(0)=s^{0} \text { et } s\left(t_{f}\right)=\bar{s} .
\end{array}\right.
$$

La résolution du problème $(\mathcal{Q})$ consiste à déterminer parmi l'infinité de trajectoires optimales en temps celle qui consomme le minimum d'oxygène et donc celle qui minimise l'énergie d'aération. 


\section{Minimisation en temps et en énergie}

Le problème en temps minimal a été résolu et on a trouvé dans la section précédente les lois de commande en oxygène qui permettent d'atteindre la cible en temps minimal. Cependant l'oxygénation du système a un coût, et ces lois minimales en temps peuvent s'avérer très coûteuses. On s'intéresse donc maintenant à des critères qui prennent en compte ce coût en pénalisant la consommation d'oxygène.

\subsection{Formulation du problème}

Dans la littérature du traitement de l'eau, la limitation par l'oxygène de la vitesse d'oxydation du carbone (respectivement de la nitrification) est généralement modélisée par une fonction de Monod en l'oxygène qui vient multiplier la fonction de croissance en carbone (respectivement en azote), d'où le nom de 'double Monod' que l'on trouve quelquefois dans les articles de modélisation. De manière analogue, l'inhibition de la réaction de dénitrification est souvent modélisée par une fonction du type '1-Monod' d'où le terme $\frac{K_{O_{2}}}{K_{O_{2}}+O_{2}}$. Supposons que les affinités des microorganismes épurateurs pour le carbone et pour l'azote sont très importantes, autrement dit, que les constantes de demi-saturation des fonctions de Monod sont faibles. Alors, lorsque la concentration en oxygène $\mathrm{O}_{2}$ va varier entre 0 (pour décrire une anoxie totale) et, à l'autre extrême, sa valeur de saturation, la fonction de Monod correspondante $\frac{\mathrm{O}_{2}}{\mathrm{~K}_{2}+\mathrm{O}_{2}}$ et son complémentaire $1-\frac{\mathrm{O}_{2}}{\mathrm{~K}_{2}+\mathrm{O}_{2}}$ vont varier entre 0 et une valeur qui sera d'autant plus proche de 1 que les constantes d'affinité sont faibles. Si on re-normalise ces fonctions en supposant maintenant que le contrôle $u$ est la fonction de Monod, on voit apparaître dans les équations les expressions du contrôle $u$ dans les équations régissant l'oxydation du carbone et/ou de la nitrification et $1-u$ dans les équations régissant la dénitrification avec le cas $u=1$ pris comme une borne supérieure vers laquelle le contrôle va tendre en cas de saturation du milieu en oxygène. On suppose donc que $\mu(u)=u$ et que la borne supérieure du contrôle est $K=u_{\max }$, dans le modèle (2.4). On obtient le modèle suivant :

$$
\left\{\begin{array}{l}
\dot{s}_{1}=-u f_{1}\left(s_{1}\right) \\
\dot{s}_{2}=-\left(u_{\max }-u\right) f_{2}\left(s_{2}\right)
\end{array}\right.
$$

Nous cherchons à minimiser un compromis entre l'énergie liée à l'oxygène injecté dans le système et la durée totale de la réaction. Ce problème se traduit mathématiquement par la résolution du problème suivant :

$$
(\mathcal{R})\left\{\begin{array}{l}
\text { Minimiser } C\left(t_{f}, u\right)=t_{f}+\alpha \int_{0}^{t_{f}} u^{2}(s) d s, \quad \alpha>0, \\
u \in L^{\infty}\left(\left[0, t_{f}\right],\left[0, u_{\max }\right]\right), \\
s \text { solution de }(4.1) \text { tel que } s(0)=s^{0} \quad \text { et } s\left(t_{f}\right) \in \mathcal{C} .
\end{array}\right.
$$

On pose dans la suite : $g_{1}\left(s_{1}^{0}\right)=\int_{a}^{s_{1}^{0}} \frac{d s}{f_{1}(s)}$ et $g_{2}\left(s_{2}^{0}\right)=\int_{b}^{s_{2}^{0}} \frac{d s}{f_{2}(s)}$, avec $s^{0} \notin \mathcal{C}$.

On note que le cas $s^{0} \in D_{1}$ est trivial dans le contexte de la minimisation d'énergie. En effet, la seule manière de joindre $s^{0}$ à la cible $\mathcal{C}$ en temps minimal est d'utiliser la phase 
anoxique qui correspond à une consommation nulle d'énergie. On limitera donc notre étude au cas où $s^{0} \in D_{2} \cup D_{3}$.

Pour $s^{0} \in D_{2} \cup D_{3}$, on étudie séparément les cas où le coût de l'énergie considérée est assez faible $\left(\alpha \leq 1 /\left(u_{\max }\right)^{2}\right)$ et le cas où le coût de l'énergie considérée est assez important $\left(\alpha>1 /\left(u_{\max }\right)^{2}\right)$. Dans le premier cas on privilégie le problème de temps minimal et dans second c'est la contrainte d'énergie qui est privilégiée.

\subsection{Trajectoires optimales pour $\alpha \leq \frac{1}{\left(u_{\max }\right)^{2}}$}

Ces trajectoires sont différentes selon que la condition initiale appartient au domaine $D_{2}$ ou $D_{3}$.

Proposition 4.1 Si $\alpha \leq 1 /\left(u_{\max }\right)^{2}$ et si $s^{0} \in D_{3}$, l'unique trajectoire optimale pour $(\mathcal{R})$ est le segment horizontal qui part de $s^{0}$.

Preuve. Si $s^{0} \in D_{3}$ et si $u$ est un contrôle admissible pour le problème $(\mathcal{R})$, alors, on peut écrire :

$$
\int_{0}^{T} u(s) d s=g_{1}\left(s_{1}^{0}\right)
$$

où $T$ est la durée pour atteindre la cible ( $T \geq \frac{g_{1}\left(s_{1}^{0}\right)}{u_{\max }}$, sinon on n'atteint pas la cible). Or l'inégalité de Cauchy - Schwartz, puis l'égalité (4.2) impliquent que :

$$
\int_{0}^{T} u^{2}(s) d s \geq \frac{\left(g_{1}\left(s_{1}^{0}\right)\right)^{2}}{T}
$$

ce qui implique que :

$$
C(T, u)=T+\alpha \int_{0}^{T} u^{2}(s) d s \geq T+\alpha \frac{\left(g_{1}\left(s_{1}^{0}\right)\right)^{2}}{T} .
$$

On considère la fonction :

$$
\begin{aligned}
\varphi:\left[\frac{g_{1}\left(s_{1}^{0}\right)}{u_{\max }},+\infty[\right. & \longrightarrow \mathbb{R} \\
T & \longrightarrow T+\alpha \frac{\left(g_{1}\left(s_{1}^{0}\right)\right)^{2}}{T}
\end{aligned}
$$

Le seul point critique de la fonction $T \longrightarrow T+\alpha \frac{\left(g_{1}\left(s_{1}^{0}\right)\right)^{2}}{T}$ est $\sqrt{\alpha} g_{1}\left(s_{1}^{0}\right)$. Comme $\sqrt{\alpha} \leq$ $\frac{1}{u_{\max }}, \varphi$ est strictement croissante et sont minimum est atteint en $T^{*}=\frac{g_{1}\left(s_{1}^{0}\right)}{u_{\max }}$. D'après l'inégalité (4.4) on déduit que pour tout $T \geq T^{*}$, on a :

$$
C(T, u) \geq C\left(T^{*}, u_{\max }\right) \text {. }
$$

On conclut donc que $u=u_{\max }$ est le contrôle optimal. La trajectoire correspondante est un segment horizontal. 
Reste à considérer le cas où les conditions initiales sont dans $D_{2}$.

Proposition 4.2 Si $\alpha \leq 1 /\left(u_{\max }\right)^{2}$ et si $s^{0} \in D_{2}$, la solution pour le problème $(\mathcal{R})$ est donnée par le contrôle $u=u_{\max } \frac{g_{1}\left(s_{1}^{0}\right)}{g_{1}\left(s_{1}^{0}\right)+g_{2}\left(s_{2}^{0}\right)}$.

Preuve. Si $s^{0} \in D_{2}$, on montre de la même façon que dans le théorème 3.1 que la trajectoire optimale, si elle existe, atteint la cible par le coin. Et d'après (4.1), on peut écrire :

$$
\int_{0}^{t_{f}} u(s) d s=g_{1}\left(s_{1}^{0}\right) \quad \text { et } \quad \int_{0}^{t_{f}}\left(u_{\max }-u(s)\right) d s=g_{2}\left(s_{2}^{0}\right),
$$

où $t_{f}$ est le temps nécessaire pour atteindre la cible. En faisant la somme de ces deux dernières équations on voit que :

$$
t_{f}=\frac{1}{u_{\max }}\left(g_{1}\left(s_{1}^{0}\right)+g_{2}\left(s_{2}^{0}\right)\right) .
$$

De plus on montre comme dans le cas précédent que pout tout $T \geq t_{f}$ on a :

$$
C(T, u)=T+\alpha \int_{0}^{T} u^{2}(s) d s \geq t_{f}+\alpha \frac{\left(g_{1}\left(s_{1}^{0}\right)\right)^{2}}{t_{f}} .
$$

D'après l'inégalité (4.5) on déduit que pour tout $T \geq t_{f}$, on a :

$$
C(T, u) \geq C\left(t_{f}, \frac{g_{1}\left(s_{1}^{0}\right)}{t_{f}}\right) .
$$

On conclut donc que le contrôle optimal existe et il vaut : $u=\frac{g_{1}\left(s_{1}^{0}\right)}{t_{f}}$.

La figure (2) présente des trajectoires optimales qui partent de $s^{0} \in D_{2}$ et qui arrivent au coin $(0.1,0.1)$ de la cible $\mathcal{C}=[0,0.1] \times[0,0.1]$

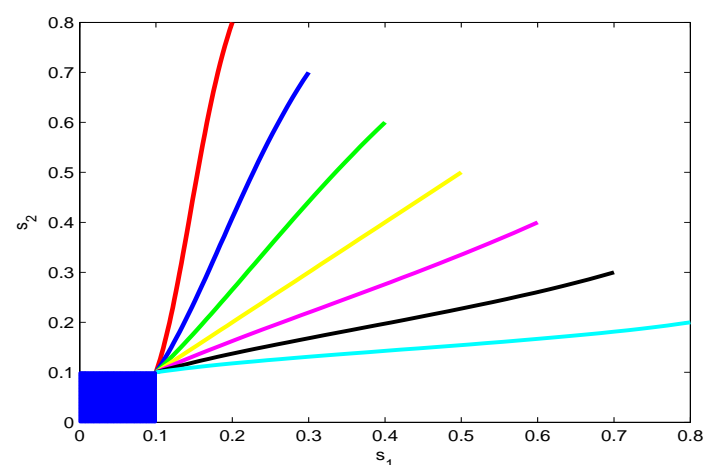

Figure 2. Exemples de trajectoires optimales qui partent de $s^{0} \in D_{2}$

Remarque 4.1 En pratique, le fait que le contrôle optimal soit constant est intéressant puisqu'il n'y a pas de manœuvre à effectuer pour injecter l'oxygène. 
Remarque 4.2 On montre que la solution du problème $(\mathcal{R})$ est aussi la solution du problème $(\mathcal{Q})$ (avec $\mu(u)=u$ ) à temps fixé. On déduit donc l'équivalence entre le problème $(\mathcal{Q})$ et le problème $(\mathcal{R})$. On peut donc résoudre le problème $(\mathcal{R})$ par une autre stratégie qui consiste à minimiser, dans un premier temps, le temps nécessaire pour atteindre la cible et déterminer, ensuite, parmi toutes ces solutions, celle qui consomme le minimum d'oxygène. On répond donc à la question posée à la fin de (3.2).

\subsection{Trajectoires optimales pour $\alpha>\frac{1}{\left(u_{\max }\right)^{2}}$}

Pour résoudre le problème $(\mathcal{R})$, on a besoin du lemme suivant :

Lemme 4.1 Soit $s^{0} \in D_{2} \cup D_{3}$. La trajectoire optimale du problème $(\mathcal{R})$, si elle existe, atteint la cible $(\mathcal{C})$ en un point qui appartient à l'axe $\left\{s_{1}=a\right\}$.

Preuve. Comme dans la preuve du théorème 3.1, on utilise le PMP. Le hamiltonien associé au problème $(\mathcal{R})$ s'écrit :

$$
H(s, p, u)=-p_{1} u f_{1}\left(s_{1}\right)-p_{2}\left(u_{\max }-u\right) f_{2}\left(s_{2}\right)+1+\alpha u^{2},
$$

avec $p_{1}$ et $p_{2}$ vérifiant (3.1), pour $\mu(u)=u$. On utilise le même raisonnement pour déduire que si $s(\cdot)$ atteint la cible en un point $s\left(t_{f}\right)$ qui appartient à $\left\{s_{2}=b\right\} \cap D_{1}$, la trajectoire $s(\cdot)$ doit être un segment vertical inclus dans $D_{1}$. Ceci est absurde puisque $s_{0} \in D_{2} \cup D_{3}$.

Proposition 4.3 Si $\alpha>1 /\left(u_{\max }\right)^{2}$ et si $s^{0} \in D_{2} \cup D_{3}$, il existe une unique trajectoire optimale solution de $(\mathcal{R})$ et elle correspond au contrôle suivant :

$$
\left\{\begin{array}{lll}
\bar{u}=u_{\max } \frac{g_{1}\left(s_{1}^{0}\right)}{g_{1}\left(s_{1}^{0}\right)+g_{2}\left(s_{2}^{0}\right)} & \text { si } & s^{0} \in\left(D_{2} \cup D_{3}\right) \backslash \Gamma, \\
\bar{u}=\frac{1}{\sqrt{\alpha}} & \text { si } & s^{0} \in \Gamma,
\end{array}\right.
$$

avec $\Gamma$ l'ensemble défini par:

$$
\Gamma=\left\{s^{0} \in D_{2} \cup D_{3} \quad \text { tel } \quad \text { que } \quad \alpha\left(u_{\max }\right)^{2}\left(g_{1}\left(s_{1}^{0}\right)\right)^{2}>\left(g_{1}\left(s_{1}^{0}\right)+g_{2}\left(s_{2}^{0}\right)\right)^{2}\right\} .
$$

(Voir Figure 3).

Preuve. Si $s^{0} \in D_{2} \cup D_{3}$, d'après le lemme (4.1), la trajectoire optimale du problème $(\mathcal{R})$ touche la cible en un point qui appartient à l'axe $\left\{s_{1}=a\right\}$, ce qui implique que :

$$
\int_{0}^{T} u(s) d s=g_{1}\left(s_{1}^{0}\right),
$$

où $T$ est le temps nécessaire pour atteindre la cible $\left(T \geq \frac{g_{1}\left(s_{1}^{0}\right)+g_{2}\left(s_{2}^{0}\right)}{u_{\max }}\right.$, sinon on n'atteint pas la cible) et $u$ un contrôle admissible.

En utilisant l'inégalité de Cauchy - Schwartz et l'égalité (4.7), on obtient :

$$
\int_{0}^{T} u^{2}(s) d s \geq \frac{\left(g_{1}\left(s_{1}^{0}\right)\right)^{2}}{T},
$$


ce qui implique que :

$$
C(T, u)=T+\alpha \int_{0}^{T} u^{2}(s) d s \geq T+\alpha \frac{\left(g_{1}\left(s_{1}^{0}\right)\right)^{2}}{T} .
$$

On considère la fonction :

$$
\begin{aligned}
\varphi:\left[\frac{g_{1}\left(s_{1}^{0}\right)+g_{2}\left(s_{2}^{0}\right)}{u_{\max }},+\infty[\right. & \longrightarrow \mathbb{R} \\
T & \longrightarrow T+\alpha \frac{\left(g_{1}\left(s_{1}^{0}\right)\right)^{2}}{T}
\end{aligned}
$$

Le seul point critique de la fonction $T \longrightarrow T+\alpha \frac{\left(g_{1}\left(s_{1}^{0}\right)\right)^{2}}{T}$ est $\sqrt{\alpha} g_{1}\left(s_{1}^{0}\right)$, alors deux cas se présentent.

- Si $s^{0} \in \Gamma$ c'est-à-dire si $\sqrt{\alpha} g_{1}\left(s_{1}^{0}\right)>\frac{g_{1}\left(s_{1}^{0}\right)+g_{2}\left(s_{2}^{0}\right)}{u_{\max }}$, le minimum de la fonction $\varphi$ est atteint en

$$
T^{*}=\sqrt{\alpha} g_{1}\left(s_{1}^{0}\right)
$$

D'après l'équation (4.9), on déduit que :

$$
C(T, u)=T+\alpha \int_{0}^{T} u^{2}(s) d s \geq C\left(\sqrt{\alpha} g_{1}\left(s_{1}^{0}\right), \frac{1}{\sqrt{\alpha}}\right) .
$$

D'ou l'existence du contrôle optimal qui vaut : $\bar{u}=\frac{1}{\sqrt{\alpha}}$.

- Si $s^{0} \in\left(D_{2} \cup D_{3}\right) \backslash \Gamma$, c'est-à-dire si $\sqrt{\alpha} g_{1}\left(s_{1}^{0}\right) \leq \frac{g_{1}\left(s_{1}^{0}\right)+g_{2}\left(s_{2}^{0}\right)}{u_{m a x}}$, le minimum de la fonction $\varphi$ est atteint en

$$
T^{*}=\frac{1}{u_{\max }}\left(g_{1}\left(s_{1}^{0}\right)+g_{2}\left(s_{2}^{0}\right)\right) .
$$

D'après l'équation (4.9), on déduit que :

$$
C(T, u)=T+\alpha \int_{0}^{T} u^{2}(s) d s \geq C\left(T^{*}, u_{\max } \frac{g_{1}\left(s_{1}^{0}\right)}{g_{1}\left(s_{1}^{0}\right)+g_{2}\left(s_{2}^{0}\right)}\right) .
$$

D'ou l'existence du contrôle optimal qui vaut : $\bar{u}=u_{\max } \frac{g_{1}\left(s_{1}^{0}\right)}{g_{1}\left(s_{1}^{0}\right)+g_{2}\left(s_{2}^{0}\right)}$.

On déduit donc que le contrôle optimal du problème $(\mathcal{R})$ existe et il est donné par :

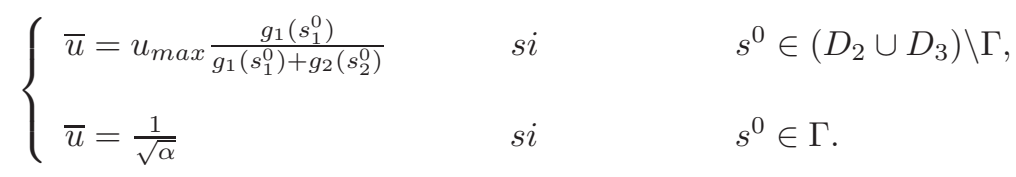


Proposition 4.4 Si $\alpha>1 /\left(u_{\max }\right)^{2}$, on $a$ :

- Si $s^{0} \in\left(D_{2} \cup D_{3}\right) \backslash \Gamma$, la trajectoire optimale touche la cible par le coin $\bar{s}=(a, b)$.

- Si s $s^{0} \in \Gamma$, la trajectoire optimale touche la cible en un point qui est situé à l'intérieur du segment $\left\{s_{1}=a\right\} \times\left\{0 \leq s_{2}<b\right\}$.

Preuve. D'après la proposition (4.3), si $s^{0} \in\left(D_{2} \cup D_{3}\right) \backslash \Gamma$, le contrôle optimal du problème $(\mathcal{R})$ est donné par $\bar{u}=u_{\max } \frac{g_{1}\left(s_{1}^{0}\right)}{g_{1}\left(s_{1}^{0}\right)+g_{2}\left(s_{2}^{0}\right)}$. Ce contrôle correspond à une trajectoire optimale qui touche la cible par le coin.

Si $s^{0} \in \Gamma, \alpha>\frac{1}{\left(u_{\max }\right)^{2}}\left(1+\frac{g_{2}\left(s_{2}^{0}\right)}{g_{1}\left(s_{1}^{0}\right)}\right)^{2}$, alors on a de façon équivalente :

$$
\frac{1}{\sqrt{\alpha}}<u_{\max } \frac{g_{1}\left(s_{1}^{0}\right)}{g_{1}\left(s_{1}^{0}\right)+g_{2}\left(s_{2}^{0}\right)} .
$$

Comme

$$
\dot{s}_{2}=-\left(u_{\max }-\frac{1}{\sqrt{\alpha}}\right) f_{2}\left(s_{2}\right)
$$

alors

$$
-\frac{\dot{s}_{2}}{f_{2}\left(s_{2}\right)}>\frac{u_{\max } g_{2}\left(s_{2}^{0}\right)}{g_{1}\left(s_{1}^{0}\right)+g_{2}\left(s_{2}^{0}\right)} .
$$

En intégrant par rapport à $t$ entre 0 et $T$, on obtient par changement de variable :

$$
\int_{s_{2}(T)}^{s_{2}^{0}} \frac{d s}{f_{2}(s)}>\frac{u_{\max } g_{2}\left(s_{2}^{0}\right)}{g_{1}\left(s_{1}^{0}\right)+g_{2}\left(s_{2}^{0}\right)} T .
$$

Or la trajectoire touche la cible par l'axe $\left\{s_{1}=a\right\}$ au temps

$$
T=\sqrt{\alpha} g_{1}\left(s_{1}^{0}\right)>\frac{1}{u_{\max }}\left(1+\frac{g_{2}\left(s_{2}^{0}\right)}{g_{1}\left(s_{1}^{0}\right)}\right) g_{1}\left(s_{1}^{0}\right)=\frac{1}{u_{\max }}\left(g_{1}\left(s_{1}^{0}\right)+g_{2}\left(s_{2}^{0}\right)\right) .
$$

Les inégalités (4.13) et (4.14) impliquent :

$$
\int_{s_{2}(T)}^{s_{2}^{0}} \frac{d s}{f_{2}(s)}>\int_{b}^{s_{2}^{0}} \frac{d s}{f_{2}(s)} .
$$

Et par suite

$$
s_{2}(T)<b
$$

La figure (3) présente des trajectoires optimales qui partent de $s^{0} \in \Gamma$ et qui arrivent à l'intérieur du segment $\left\{s_{1}=0.1\right\} \times\left\{0 \leq s_{2} \leq 0.1\right\}$ et une trajectoire optimale qui part de $s^{0} \in\left(D_{2} \cup D_{3}\right) \backslash \Gamma$ et qui arrive sur le coin de la cible $\mathcal{C}=[0,0.1] \times[0,0.1]$. 


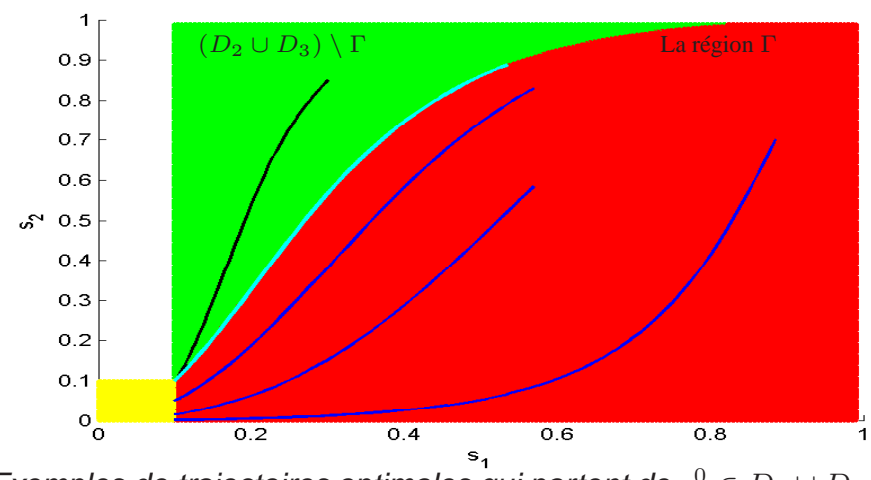

Figure 3. Exemples de trajectoires optimales qui partent de $s^{0} \in D_{2} \cup D_{3}$

Remarque 4.3 En conclusion, si $\alpha$ est assez petit, le temps final est le temps minimal obtenu dans la section 3. Mais, quand $\alpha$ est assez grand, en partant d'une condition initiale $s^{0} \in \Gamma$, l'énergie est minimale mais la trajectoire met plus de temps pour atteindre la cible. En revanche, pour $s^{0} \notin \Gamma$, l'énergie de l'oxygène dépensé est plus grande et le temps est minimal.

Ainsi, quand le coût lié à l'oxygénation est relativement important (par rapport au coût lié au temps), il peut arriver que la stratégie optimale ne minimise pas le temps. Dans ce cas, la concentration finale de l'un des substrats $\left(s_{2}\right)$ sera inférieure au seuil de tolérance.

Remarque 4.4 Dans le cas où $\alpha=\frac{1}{\left(u_{\max }\right)^{2}}$, les résultats de la section 4.2 coüncident avec ceux de 4.3.

\section{Conclusion}

Dans ce travail, on s'intéresse au contrôle d'un bioprocédé séquentiel discontinu pour le traitement de la pollution dans les eaux usées. On a considéré un schéma réactionnel faisant intervenir deux réactions distinctes. L'une de ces deux réactions est supposée être aérobie tandis que l'autre est anoxique. On s'est intéressé au problème de contrôle optimal où la variable de contrôle est la concentration en oxygène dissous.

Dans un premier temps, on a étudié un problème de contrôle en temps minimal. On a prouvé qu'il existe soit une unique trajectoire optimale, soit une infinité de trajectoires optimales selon la valeur de la condition initiale des concentrations des deux substrats dans le réacteur.

Ensuite, on a étudié un autre problème de contrôle optimal où la fonction coût est un compromis entre le temps de réaction et l'énergie liée à l'aération. On a montré que si la proportion de l'énergie considérée est assez faible $\left(\alpha \leq 1 /\left(u_{\max }\right)^{2}\right)$, il existe une unique 
trajectoire optimale. On a un résultat analogue si on commence par minimiser d'abord le temps ainsi que proposé dans la première partie du travail puis l'énergie. En revanche, si la proportion de l'énergie considérée est assez importante $\left(\alpha>1 /\left(u_{\max }\right)^{2}\right)$, la stratégie du cas précédent n'est plus optimale. Dans ce cas, on a montré qu'il existe une unique trajectoire optimale qui dépend de la condition initiale des concentrations en substrats. Dans la suite des travaux, on compte s'intéresser à un modèle plus proche de la réalité en incorporant un substrat limitant supplémentaire afin de résoudre le problème pour un système impliquant à la fois des composés azotés et de la matière organique carbonée. A notre connaissance, c'est une des premières modélisations où sont explicités les influences opposés de l'oxygène sur les deux réactions biologiques : à ce stade, nous sommes conscients qu'il s'agit d'une approche simplificatrice. Nous comptons développer ultérieurement l'étude d'une prise en compte plus fine, par exemple en considérant que le contrôle n'est plus l'oxygène mais sa dérivée, ce qui est plus réaliste.

Remerciements : Les auteurs remercient le projet TREASURE (projet INRIA Euromed 3+3) pour le soutien apporté à ce travail.

\section{Bibliographie}

[1] D. Mazouni, Thèse de doctorat - Modélisation et commande en temps minimum des réacteurs biologiques séquentiels discontinus, Université Claude Bernard-Lyon I, (2006).

[2] J. Moreno, Optimal time control of bioreactors for the wastewater treatment. Optim. Control Appl. Meth., No 20, p 145-164, (1999).

[3] D. Mazouni, J. Harmand, A. Rapaport and H. Hammouri, Optimal time control of switching systems : application to biological Sequencing Batch Reactors, Optimal Control Application and Methods, Vol. 31, No.4, pp. 289-301. (2010).

[4] M. Henze, W. Gujer, T. Mino, M. van Loosdrecht, Activated sludge models ASM1, ASM2, ASM2d and ASM3, Edited by IWA Task group on mathematical modelling for design and operation of biological wastewater treatment, IWA Publishing, 2000.

[5] E.TRÉLAT, Contrôle optimal, théorie et applications. Vuibert, collection "Mathématiques concrètes", (2005), 173.

[6] L. Pontryagin and V. Boltyanskit and R. Gamkrelidze and E. Mischenko, The Mathematical Theory of Optimal Processes. Wiley Interscience (1962), p 159-160, Edition de l'école polytechnique. Juillet 2006.

[7] F.Bonnans, P.Rouchon, Analyse et commande de systèmes dynamiques, Edition de l'école polytechnique. (2003). 